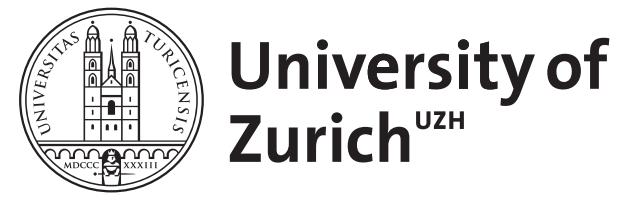

Zurich Open Repository and Archive

University of Zurich

University Library

Strickhofstrasse 39

CH-8057 Zurich

www.zora.uzh.ch

Year: 2020

\title{
Proper case selection and assessment
}

Papageorgiou, Spyridon N

DOI: https://doi.org/10.1177/1465312520923673

Posted at the Zurich Open Repository and Archive, University of Zurich

ZORA URL: https://doi.org/10.5167/uzh-194612

Journal Article

Accepted Version

Originally published at:

Papageorgiou, Spyridon N (2020). Proper case selection and assessment. Journal of Orthodontics, 47(2):185-186. DOI: https://doi.org/10.1177/1465312520923673 


\section{TITLE PAGE}

Proper case selection and assessment

Spyridon N. Papageorgiou1

1 Clinic of Orthodontics and Pediatric Dentistry, Center of Dental Medicine, University of Zurich, Zurich, Switzerland

Corresponding author: Spyridon N Papageorgiou, Clinic of Orthodontics and Pediatric Dentistry, Center of Dental Medicine, University of Zurich, Plattenstrasse 11, Zurich $\mathrm{CH}$ 8032, Switzerland.

Email: snpapage@gmail.com.

Declaration of conflicting interests

The author declared no potential conflicts of interest with respect to the research, authorship, and/or publication of this article.

Funding

The author received no financial support for the research, authorship, and/or publication of this article.

ORCID iD

Spyridon N Papageorgiou https://orcid.org/0000-0003-1968-3326 
Theoretical scenario

Two different clinical scenarios are provided regarding the influence of orthodontic treatment with fixed appliances on the development of gingival recession.

\section{Scenario A}

Harry is an orthodontist who runs a busy practice with lots of new cases being started each week. Making a retrospective check of his charts, he identifies that over the last five years, 13 patients have reported back to his practice at least three years after completion of fixed appliance treatment with a chief complaint of sensitivity to cold. Harry had asked the patients to localise the sensitivity within their mouth and by examining those teeth saw that $11 / 13(85 \%)$ patients had a recession of the labial gingiva of a mandibular incisor, which might contribute to the patients' sensitivity to cold. Estimating that he has a turnaround of about 1000 patients actively being treated within the observation period of five years, Harry boasts that he has only a miniscule $1 \%(11 / 1000)$ of patients with gingival recession in his orthodontic practice.

\section{Scenario B}

After hearing this story, Oliver, a friend and colleague of Harry, vaguely remembers that he has had similar calls to his own practice by former patients of his and is worried he might have overlooked the risk of gingival recession in his practice. He searches his patient archives for similar calls from 25 debonded patients complaining of tooth sensitivity in the last five years and calls them back to the practice for a thorough clinical examination. Upon close examination of all teeth, he finds more than 20 of the 25 patients with at least one gingival recession of a mandibular incisor. Interestingly, he also finds many recessions on the labial gingiva of not only mandibular incisors but also of other posterior teeth in both jaws and finds that not all recessions are associated with a sensitivity to cold. Furthermore, he even finds that some of the identified recessions are on the palatal/lingual aspects of the teeth. Feeling he might have missed a big part of the clinical picture of recessions, he uses a computer-based random number generator to invite for a free clinical examination a random sample of 300 patients that were debonded in the last five years, regardless of whether they had any symptoms (such as sensitivity to cold). To his astonishment, he finds that over $70 \%$ of his patients had at least one gingival recession of anterior or posterior teeth and these on either the labial and/or the palatal/ lingual side of the teeth. He feels disheartened by the high percentage of gingival recession that comes as a result of orthodontic treatment, but at least he feels his is a much more honest evaluation than Harry's.

Which of the following statements are correct, if any?

(a) Scenario A provides a trustworthy estimate of the general prevalence of gingival recession;

(b) Scenario B provides a trustworthy estimate of the general prevalence of gingival recession; or

(c) Scenario B adequately estimates the contribution of orthodontic treatment in the development of gingival recessions.

\section{Discussion}


One good starting point is to critically analyse the patient sample that was tested for gingival recession in each of the scenarios. Scenario A included cases with symptoms reported by the patients. While we can see that the symptom of sensitivity to cold stimuli has a relatively high positive predictive value for posttreatment gingival recessions (85\%; 11 out of 13 patients with sensitivity also had a recession), this does not mean that it is all-inclusive. Indeed, there can be patients without any symptoms (here, cold sensitivity) who also have the condition in question (here, gingival recession). Which means that if only patients with clinical symptoms are examined, then the measured number will not reflect the average percentage of patients with recessions, since not all recessions might be associated with sensitivity to cold. Furthermore, Harry examined clinically only those teeth the patient pointed out as symptomatic. This is problematic, since laypersons might lack the training and critical eye to identify changes in the position, colour or texture of soft tissues, especially when these are of small magnitude. Therefore statement (a) is false, since both the sample assessed was not representative of the average patient treated orthodontically and issues existed in the outcome assessment protocol.

Scenario $B$ is a considerable improvement in sample size representativeness. Oliver chose to draw a completely random sample of patients from the pool of patients debonded in his practice in the last five years. Provided that the chosen sample size is not very small, the random patient selection is bound to provide a less biased sample that might be more generalisable to the average orthodontic patient. Furthermore, the protocol he chose to ascertain the existence of gingival recessions is much more thorough, since both anterior and posterior regions of both jaws were examined from both labial and palatal/lingual surfaces. This reduces the number of 'false negatives' or, in other terms, reduces the risk of labelling a patient recession-free, even though the patient has at least one recession. Therefore, scenario B provides a much more representative estimate of the prevalence of (any) gingival recessions among patients having been treated orthodontically with fixed appliances and statement (b) is correct.

However, whether these cases of gingival recession can be attributed to the orthodontic treatment is another matter. For one, gingival recession can also be found among orthodontic patients before orthodontic treatment (Gebistorf et al., 2018). So, in order to come closer to the true effect of orthodontic treatment on the development of gingival recessions, we need to move away from the point prevalence of gingival recessions (number of patients with recession divided by total number of patients) towards the incidence proportion of gingival recessions. Contrary to the prevalence, which counts both pre-existing and new cases, the incidence counts only new cases of disease (and divides them by the total number of patients); therefore, this is closer to the additive burden of recessions attributed to orthodontic treatment. Furthermore, gingival recessions can also be found among people who have not been treated orthodontically (Renkema et al., 2013), so a comparative group of untreated patients needs to be used. Additionally, even among untreated patients, the prevalence of gingival recessions increases with age (Renkema et al., 2013), which means that just finding a group of untreated patients and taking a 'snapshot' measurement of their recessions is not enough and a longitudinal assessment is needed. Therefore, the ideal study to investigate the effect of orthodontic treatment would measure the incidence of new recessions and not the prevalence in a longitudinal manner and include a control group of untreated patients. Finally, 
since pre-treatment patient characteristics (such as pre-existing recession of a neighbouring tooth or crowding coupled with a thin biotype) might be potential confounders, ideally a randomized clinical trial would be employed to ensure baseline similarity between treated and untreated patients. And as scenario $B$ resembles a cross-sectional study on the prevalence of solely treated patients, statement (c) is false.

References

Gebistorf M, Mijuskovic M, Pandis N, Fudalej PS and Katsaros C (2018). Gingival recession in orthodontic patients 10 to 15 years posttreatment: A retrospective cohort study. American Journal of Orthodontics and Dentofacial Orthopedics 153: 645-655.

Renkema AM, Fudalej PS, Renkema AA, Abbas F, Bronkhorst E and Katsaros C (2013) Gingival labial recessions in orthodontically treated and untreated individuals: a case - control study. Journal of Clinical Periodontology 40: 631-637. 